PROCEEDINGS OF THE

AMERICAN MATHEMATICAL SOCIETY

Volume 131, Number 1, Pages 141-146

S 0002-9939(02)06528-0

Article electronically published on May 9, 2002

\title{
THE COMPOSITION OF PROJECTIONS ONTO CLOSED CONVEX SETS IN HILBERT SPACE IS ASYMPTOTICALLY REGULAR
}

\author{
HEINZ H. BAUSCHKE
}

(Communicated by Jonathan M. Borwein)

\begin{abstract}
The composition of finitely many projections onto closed convex sets in Hilbert space arises naturally in the area of projection algorithms. We show that this composition is asymptotically regular, thus proving the socalled "zero displacement conjecture" of Bauschke, Borwein and Lewis. The proof relies on a rich mix of results from monotone operator theory, fixed point theory, and convex analysis.
\end{abstract}

\section{THE PROBLEM}

We assume that

$$
X \text { is a real Hilbert space with inner product }\langle\cdot, \cdot\rangle \text {, }
$$

and that

$$
C_{1}, \ldots, C_{N} \text { are closed convex nonempty sets in } X
$$

with

$$
\text { corresponding projections } P_{1}, \ldots, P_{N} \text {. }
$$

Our aim is to show that the composition $P_{N} P_{N-1} \cdots P_{1}$ is asymptotically regular 7], i.e.,

$$
\left(P_{N} P_{N-1} \cdots P_{1}\right)^{k} x-\left(P_{N} P_{N-1} \cdots P_{1}\right)^{k+1} x \rightarrow 0, \quad \text { for every } x \in X .
$$

This in turn will imply the "zero displacement conjecture", formulated in 4].

We briefly sketch the origin and the interest in this conjecture. (The reader is referred to [4, 2] and [9] and the various references therein for further comments.) Numerous problems in mathematics and physical sciences can be recast as a convex feasibility problem: find $x \in \bigcap_{n=1}^{N} C_{n}$. A well-known result due to Bregman states that the sequence of iterates $\left(P_{N} \cdots P_{1}\right)^{k} x$ converges weakly to a point in the intersection provided it is nonempty [5. However, in applications it may not be a priori clear whether or not the intersection is nonempty. Hence, one wishes clarity about the asymptotic behaviour of the sequence in the inconsistent case. A complete analysis has been carried out for $N=2$ or when each set $C_{n}$ is an affine subspace. Nonetheless, even for $N=3$, some nagging questions on the behavior of

Received by the editors February 20, 2001 and, in revised form, August 13, 2001.

2000 Mathematics Subject Classification. Primary 47H05, 47H09, 90C25.

The author's research was supported by NSERC. 
the sequence remain. We believe that the asymptotic regularity established here is an important step towards a complete understanding of the sequence $\left(P_{N} \cdots P_{1}\right)^{k} x$.

It will be extremely convenient to work in the product Hilbert space $X^{N}$, equipped with the induced inner product

$$
\langle x, y\rangle=\sum_{n=1}^{N}\left\langle x_{n}, y_{n}\right\rangle, \quad \text { for all } x=\left(x_{n}\right)_{n=1}^{N} \text { and } y=\left(y_{n}\right)_{n=1}^{N} \text { in } X^{N}
$$

We use standard notation and results from convex analysis and monotone operator theory; see, for instance, [1] and [13.

\section{Preliminaries}

We define the "diagonal"

$$
\Delta:=\left\{(\xi, \xi, \ldots, \xi) \in X^{N}: \xi \in X\right\}
$$

and the right-shift operator

$$
R: X^{N} \rightarrow X^{N}:\left(x_{1}, x_{2}, \ldots, x_{N}\right) \mapsto\left(x_{N}, x_{1}, \ldots, x_{N-1}\right) .
$$

Clearly, $R$ is an isometry and the conjugate (or adjoint) of $R$ is the left-shift operator

$$
L: X^{N} \rightarrow X^{N}:\left(x_{1}, x_{2}, \ldots, x_{N}\right) \mapsto\left(x_{2}, x_{3}, \ldots, x_{N}, x_{1}\right) .
$$

Now set

$$
M=I-R \text {. }
$$

Proposition 2.1. $M$ is maximal monotone.

Proof. Clearly, $M$ is a continuous linear operator from $X^{N}$ to itself. For $x \in X^{N}$, we have $\langle x, R x\rangle \leq\|x\|\|R x\|=\|x\|\|x\|=\langle x, x\rangle$, and hence $\langle x, M x\rangle=\langle x,(I-R) x\rangle \geq 0$. By linearity of $M$, the operator $M$ is monotone. Continuity now implies maximal monotonicity; see, for instance, [12, Corollary 2.6].

Fact 2.2. Suppose $A$ is a continuous linear monotone operator from a Banach space $E$ to its dual $E^{*}$. Let $S=\frac{1}{2} A+\frac{1}{2} A^{*}$ be the symmetric part of $A$, where $A^{*}$ denotes the conjugate operator of $A$. Further, let $q(x)=\frac{1}{2}\langle x, A x\rangle$, for all $x \in E$. Then:

(i) $q$ is convex and Gâteaux differentiable with $\nabla q=S$.

(ii) $q^{*} \circ S=q$, where $q^{*}$ is the conjugate function of $q$ from convex analysis.

(iii) $\operatorname{ran} S \subseteq \operatorname{dom} q^{*} \subseteq \operatorname{cl} \operatorname{ran} S$.

Proof. For (i) and (ii), see [3, Theorem 3.6.(i)].

(iii): this is in [1, Proposition 12.3.6.(iii)]; for the reader's convenience, we repeat the argument here. Note first that (ii) implies the inclusion $\operatorname{ran} S \subseteq \operatorname{dom} q^{*}$. Next, fix $x^{*} \in \operatorname{dom} q^{*}$ and let $f=q-x^{*}$. Then

$$
q^{*}\left(x^{*}\right)=\sup _{x \in E}\left[\left\langle x^{*}, x\right\rangle-q(x)\right]=-\inf _{x \in E}\left[q(x)-\left\langle x^{*}, x\right\rangle\right]=-\inf _{x \in E} f(x) .
$$

Fix $\epsilon>0$. By [11, Lemma 3.22], there exists some $x \in E$ such that $\|\nabla f(x)\|=$ $\left\|S x-x^{*}\right\|<\epsilon$. Since $\epsilon$ was chosen arbitrarily, it follows that $x^{*} \in \operatorname{cl} \operatorname{ran} S$. 
We now let $S$ be the symmetric part of $M$, i.e.,

$$
S=\frac{1}{2} M+\frac{1}{2} M^{*}=I-\frac{1}{2} R-\frac{1}{2} L
$$

and

$$
\left.\left.q: X^{N} \rightarrow\right]-\infty,+\infty\right]: x \mapsto \frac{1}{2}\langle x, M x\rangle .
$$

Proposition 2.3. $\operatorname{ran} S=\operatorname{dom} q^{*}=\Delta^{\perp}$.

Proof. In light of Proposition 2.1 and Fact 2.2(iii), we have $\operatorname{ran} S \subseteq \operatorname{dom} q^{*} \subseteq$ clran $S$. Thus it suffices to show that $\operatorname{ran} S=\Delta^{\perp}$. It is easy to see that $y=$ $\left(y_{n}\right)_{n=1}^{N} \in \Delta^{\perp}$ if and only if $\sum_{n=1}^{N} y_{n}=0$. Using this, it is readily verified that ran $S \subseteq \Delta^{\perp}$. The reverse inclusion takes a little more work: pick $y=\left(y_{n}\right)_{n=1}^{N} \in \Delta^{\perp}$. We need to show that $y \in \operatorname{ran} S$. For $1 \leq n \leq N-1$, set

$$
z_{n}=y_{1}+2 y_{2}+3 y_{3}+\cdots+n y_{n} .
$$

Next, set $x_{N}=0$ and define $x=\left(x_{n}\right)_{n=1}^{N}$ by the backwards recursion

$$
(n+1) x_{n}-n x_{n+1}=z_{n}, \quad \text { for } 1 \leq n \leq N-1 .
$$

Then $y_{1}=z_{1}=2 x_{1}-x_{2}$ and thus

$$
-x_{N}+2 x_{1}-x_{2}=y_{1} .
$$

On the other hand, $n y_{n}=z_{n}-z_{n-1}=(n+1) x_{n}-n x_{n+1}-n x_{n-1}+(n-1) x_{n}=$ $2 n x_{n}-n x_{n+1}-n x_{n-1}$, or

$$
-x_{n-1}+2 x_{n}-x_{n+1}=y_{n}, \quad \text { for } 2 \leq n \leq N-1 .
$$

Altogether, $\sum_{n=1}^{N-1} y_{n}=x_{1}+x_{N-1}-2 x_{N}$. This implies, using $\sum_{n=1}^{N} y_{n}=0$ or equivalently $\sum_{n=1}^{N-1} y_{n}=-y_{N}$,

$$
-x_{N-1}+2 x_{N}-x_{1}=y_{N} .
$$

The last three displayed equations are equivalent to $y=S(2 x)$.

The following fact on the approximation of the sum of two monotone operators will be crucial.

Fact 2.4 (Brézis-Haraux). Suppose $E$ is a Hilbert space, and $S_{1}$ and $S_{2}$ are two monotone operators from $E$ to $2^{E^{*}}$ such that $S_{1}+S_{2}$ is maximal monotone. Suppose further that $\operatorname{dom} S_{1} \subseteq \operatorname{dom} S_{2}$, and for every $x_{2} \in \operatorname{dom} S_{2}$ and $y_{2}^{*} \in \operatorname{ran} S_{2}$, we have

$$
\sup _{w^{*} \in S_{2}(w)}\left\langle w-x_{2}, y_{2}^{*}-w^{*}\right\rangle<+\infty
$$

Then:

(i) $\operatorname{cl} \operatorname{ran}\left(S_{1}+S_{2}\right)=\operatorname{cl}\left(\operatorname{ran}\left(S_{1}\right)+\operatorname{ran}\left(S_{2}\right)\right)$, and

(ii) int $\operatorname{ran}\left(S_{1}+S_{2}\right)=\operatorname{int}\left(\operatorname{ran}\left(S_{1}\right)+\operatorname{ran}\left(S_{2}\right)\right)$.

Proof. See [6] as well as the new approach in [13, Section 19].

We conclude this section with an immensely useful result from fixed point theory. Recall that a map $T$ is called strongly nonexpansive [8], if it is nonexpansive, and $\left(x_{k}-y_{k}\right)-\left(T x_{k}-T y_{k}\right) \rightarrow 0$ whenever $\left(x_{k}-y_{k}\right)$ is bounded and $\left\|x_{k}-y_{k}\right\|-\| T x_{k}-$ $T y_{k} \| \rightarrow 0$ for sequences $\left(x_{k}\right),\left(y_{k}\right)$. 
Fact 2.5 (Bruck-Reich). Suppose $E$ is a Hilbert space, and $T: E \rightarrow E$ is strongly nonexpansive. Then there exists a vector $v \in E$ such that $T^{k} x-T^{k+1} x \rightarrow v$. In fact, $v$ is the unique element of minimum norm in $\operatorname{cl} \operatorname{ran}(I-T)$.

Proof. This follows from the considerably more general [8, Corollary 1.5]. (The function $\frac{1}{2}\|\cdot\|^{2}$ is Fréchet differentiable in Hilbert space, and $E$ is a sunny nonexpansive retract of itself (via the identity); hence Bruck and Reich's assumptions in [8, Corollary 1.5] are indeed satisfied.)

\section{MAin RESUlt}

Theorem 3.1. The composition $\left(P_{N} P_{N-1} \cdots P_{1}\right)$ is asymptotically regular.

Proof. We work mostly in the product space $X^{N}$, in which we set $C=C_{1} \times \cdots \times C_{N}$. Separability of the set $C$ readily implies that $P_{C}$, the projection onto $C$, is separable as well: $P_{C}=P_{1} \times \cdots \times P_{N}$. Denote the subdifferential map of the indicator function of $C$ by $N_{C}$. We now proceed in several steps.

Step 1: $N_{C}$ is maximal monotone.

This is a consequence of Rockafellar's maximal monotonicity theorem; see, for instance, [11, Theorem 3.24].

Step 2: $N_{C}+M$ is maximal monotone.

By Step 1, $N_{C}$ is maximal monotone. On the other hand, $M$ is maximal monotone (Proposition 2.1) with $\operatorname{dom} M=X^{N}$. Altogether, by Rockafellar's sum theorem [13, Section 20], $N_{C}+M$ is maximal monotone.

Step 3: $\sup _{w \in X^{N}}\langle w-x, M y-M w\rangle<+\infty$, for all $x, y \in X^{N}$.

Using Proposition 2.3 and $\left(M y+M^{*} x\right) / 2 \in \Delta^{\perp}$, we indeed have

$$
\begin{aligned}
\sup _{w}\langle w-x, M y-M w\rangle & =-\langle x, M y\rangle+2 \sup _{w}\left[\left\langle w, \frac{1}{2} M y+\frac{1}{2} M^{*} x\right\rangle-q(w)\right] \\
& =-\langle x, M y\rangle+2 q^{*}\left(\frac{1}{2} M y+\frac{1}{2} M^{*} x\right) \\
& <+\infty .
\end{aligned}
$$

Step 4: $\operatorname{cl} \operatorname{ran}\left(M+N_{C}\right)=\operatorname{cl}\left(\operatorname{ran}(M)+\operatorname{ran}\left(N_{C}\right)\right)$.

Let $S_{1}=N_{C}$ and $S_{2}=M$. Then $S_{1}$ and $S_{2}$ are both maximal monotone (Step 1 and Proposition 2.1), and so is their sum (Step 2). Also, $\operatorname{dom} S_{1} \subseteq \operatorname{dom} S_{2}$. Now the desired equality follows from Step 3 and Fact 2.4

Step 5: $0 \in \operatorname{cl} \operatorname{ran}\left(M+N_{C}\right)$.

Clearly, $0 \in \operatorname{ran}(M) \cap \operatorname{ran}\left(N_{C}\right)$. Hence $0 \in \operatorname{ran}(M)+\operatorname{ran}\left(N_{C}\right)$. By Step 4, we obtain $0 \in \operatorname{cl} \operatorname{ran}\left(M+N_{C}\right)$.

Step 6: $(\forall \epsilon>0)\left(\exists b \in X^{N}\right)(\exists x \in C):\|b\| \leq \epsilon$ and $x=P_{C}(R x+b)$.

Indeed, fix $\epsilon>0$. By Step 5, we obtain $x \in X^{N}$ and $b \in M(x)+N_{C}(x)$ with $\|b\| \leq \epsilon$. Hence $x \in C$. Thus $b \in M(x)+N_{C}(x) \Leftrightarrow(R x+b)-x \in N_{C}(x) \Leftrightarrow$ $\sup \langle C-x,(R x+b)-x\rangle \leq 0 \Leftrightarrow x=P_{C}(R x+b)$.

Step 7: $(\forall \epsilon>0)\left(\exists d \in X^{N}\right)(\exists x \in C):\|d\| \leq \epsilon$ and $x=P_{C}(R x)+d$.

This follows from Step 6 and the fact that $P_{C}$ is nonexpansive.

Step 8: $(\forall \epsilon>0)(\exists x \in C)(\forall 1 \leq n \leq N)$ :

$$
\left\|P_{n-1} \cdots P_{1} x_{N}-P_{n} P_{n-1} \cdots P_{1} x_{N}-x_{n-1}+x_{n}\right\| \leq(2 n-1) \epsilon
$$


where $x_{0}=x_{N}$. Fix $\epsilon>0$ and obtain $x$ and $d$ as in Step 7. Let $1 \leq n \leq N$. Then $x_{n}=P_{n} x_{n-1}+d_{n}$. Now $P_{n}$ is nonexpansive, and $\left\|d_{n}\right\| \leq\|d\| \leq \epsilon$, hence

$$
\begin{aligned}
\left\|P_{n} P_{n-1} \cdots P_{1} x_{0}-x_{n}\right\| & \leq\left\|P_{n} P_{n-1} \cdots P_{1} x_{0}-P_{n} x_{n-1}\right\|+\left\|d_{n}\right\| \\
& \leq\left\|P_{n-1} \cdots P_{1} x_{0}-x_{n-1}\right\|+\epsilon .
\end{aligned}
$$

It follows by induction that, if $1 \leq n \leq N$, then

$$
\left\|P_{n} P_{n-1} \cdots P_{1} x_{0}-x_{n}\right\| \leq n \epsilon .
$$

Adding $(n)$ and $(n-1)$, followed by an application of the triangle inequality, completes the proof of Step 8.

Step 9: $(\forall \epsilon>0)(\exists \xi \in X):\left\|\xi-\left(P_{N} P_{N-1} \cdots P_{1}\right)(\xi)\right\| \leq N^{2} \epsilon$.

For fixed $\epsilon>0$, obtain $x \in C$ as in Step 8. For $1 \leq n \leq N$, set $e_{n}=$ $P_{n-1} \cdots P_{1} x_{N}-P_{n} P_{n-1} \cdots P_{1} x_{N}-x_{n-1}+x_{n}$ so that $\left\|e_{n}\right\| \leq(2 n-1) \epsilon$. Let $\xi=x_{N}$. On the one hand, $\sum_{n=1}^{N} e_{n}$ telescopes to $\xi-\left(P_{N} P_{N-1} \cdots P_{1}\right)(\xi)$. On the other hand, $\epsilon(1+3+5+\cdots+(2 N-1))=N^{2} \epsilon$. Altogether, Step 9 follows.

Last Step: $P_{N} P_{N-1} \cdots P_{1}$ is asymptotically regular.

For brevity, denote this composition by $T$. It is well-known that projections are firmly nonexpansive; see, for instance, [10, Chapter 12]. Hence each $P_{n}$ is strongly nonexpansive [8, Proposition 2.1], and so is the composition $T$ [8, Proposition 1.1]. In view of Step 9 , we have $0 \in \operatorname{cl} \operatorname{ran}(I-T)$. The asymptotic regularity of $T$ now follows from Fact 2.5

Remark 3.2. It is easy to see that the "zero displacement conjecture" 4 Conjecture 5.3.6] is equivalent to the asymptotic regularity of $P_{N} \cdots P_{1}$.

\section{ACKNOWLEDGMENT}

I wish to thank an anonymous referee for suggestions yielding considerably cleaner proofs.

\section{REFERENCES}

[1] H. H. Bauschke. Projection algorithms and monotone operators. Ph.D. thesis, Simon Fraser University, 1996. Available at http://www.cecm.sfu.ca/preprints/1996pp.html.

[2] H. H. Bauschke and J. M. Borwein. On projection algorithms for solving convex feasibility problems. SIAM Review, 38:367-426, 1996. MR 98f:90045

[3] H. H. Bauschke and J. M. Borwein. Maximal monotonicity of dense type, local maximal monotonicity, and monotonicity of the conjugate are all the same for continuous linear operators. Pacific Journal of Mathematics, 189(1):1-20, 1999. MR 2001j:47059

[4] H. H. Bauschke, J. M. Borwein, and A. S. Lewis. The method of cyclic projections for closed convex sets in Hilbert space. In Recent developments in optimization theory and nonlinear analysis (Jerusalem, 1995), pages 1-38. Amer. Math. Soc., Providence, RI, 1997. MR 98c:49069

[5] L. M. Bregman. The method of successive projection for finding a common point of convex sets. Soviet Math. Dokl., 6:688-692, 1965.

[6] H. Brézis and A. Haraux. Image d'une somme d'opérateurs monotones et applications. Israel Journal of Mathematics, 23(2):165-186, 1976. MR 53:3803

[7] F. E. Browder and W. V. Petryshin. The solution by iteration of nonlinear functional equations in Banach spaces. Bulletin of the American Mathematical Society, 72:571-575, 1966. MR 32:8155b

[8] R. E. Bruck and S. Reich. Nonexpansive projections and resolvents of accretive operators in Banach spaces. Houston Journal of Mathematics, 3(4):459-470, 1977. MR 57:10507 
[9] A. R. De Pierro. From parallel to sequential projection methods and vice versa in convex feasibility: results and conjectures. In D. Butnariu, Y. Censor, and S. Reich (editors) Inherently Parallel Algorithms in Feasibility and Optimization and their Applications (Haifa, 2000), pages 187-202, Elsevier 2001.

[10] K. Goebel and W. A. Kirk. Topics in metric fixed point theory. Cambridge University Press, 1990. MR 92c: 47070

[11] R. R. Phelps. Convex functions, monotone operators and differentiability. Springer-Verlag, Berlin, second edition, 1993. MR 94f:46055

[12] R. R. Phelps and S. Simons. Unbounded Linear Monotone Operators on Nonreflexive Banach Spaces. Journal of Convex Analysis, 5(2):303-328, 1998. MR 99k:47003

[13] Stephen Simons. Minimax and monotonicity. Springer-Verlag, Berlin, 1998. MR 2001h: 49002

Department of Mathematics and Statistics, University of Guelph, Guelph, Ontario, Canada N1G 2W1

E-mail address: hbauschk@uoguelph.ca 